Revisión

\title{
Indicaciones y resultados a largo plazo de los trasplantes de órganos sólidos. Calidad de vida en pacientes trasplantados
}

\author{
D. DAGA RUIZa , C. FERNÁNDEZ AGUIRRE ${ }^{b}$, F. SEGURA GONZÁLEZª Y M. CARBALLO RUIZa \\ aServicio de Cuidados Críticos y Urgencias. Coordinación de Trasplantes. \\ bervicio de Neumología. Hospital Universitario Virgen de la Victoria. Málaga. España.
}

El trasplante de órganos constituye uno de los avances más espectaculares en la Historia de la Medicina. Los trasplantes de órganos, que todavía eran un reto lejano en la década de los setenta del siglo pasado, constituyen actualmente un procedimiento rutinario de la práctica médica que ha contribuido a prolongar la supervivencia y la calidad de vida de la población.

España ha alcanzado la tasa más elevada del mundo en donación y trasplantes por millón de habitantes, siendo esta extraordinaria tarea asistencial un logro del sistema sanitario español en su conjunto.

El objetivo de esta revisión es repasar las indicaciones de los trasplantes de órganos sólidos en la actualidad y sus resultados a largo plazo, evaluando el impacto sobre la calidad de vida relacionada con la salud de los pacientes receptores de un trasplante de órgano sólido.

PALABRAS CLAVE: trasplante de órganos, indicaciones y resultados, calidad de vida.

INDICATIONS AND LONG-TERM OUTCOMES FOR SOLID ORGAN TRANSPLANT. QUALITY OF LIFE IN SOLID ORGAN TRANSPLANT RECIPIENTS

Organ transplant constitutes one of the most encouraging advances in the history of the medi-

Correspondencia: Dr. D. Daga Ruiz.

UCI Hospital Virgen de la Victoria.

Campus Teatinos, s/n.

29010 Málaga. España.

Correo electrónico: domingo.daga.sspa@juntadeandalucia.es

Manuscrito aceptado el 11-II-2008. cine. The organ transplants that were a distant challenge in the seventies currently are a routine procedure in the medical practice that has contributed to extending survival and quality of life in the general population.

Spain has reached the highest rate for donation and transplants per one million inhabitants worldwide, this extraordinary health care work being a combined achievement of Spanish Health Care System.

The objective of this article is to review the indications of solid organ transplants and their long-term outcomes, evaluating the impact on the health-related quality of life in solid organ transplant recipients.

KEY WORDS: organ transplant, indications, outcome, quality of life.

\section{INTRODUCCIÓN}

Los trasplantes de órganos humanos permiten salvar muchas vidas y restaurar las funciones esenciales en circunstancias en que no existe ninguna otra alternativa médica de eficacia comparable.

Los trasplantes de órganos sólidos, como riñones, hígado, corazón, páncreas o pulmones son cada vez más un componente sistemático de la atención de salud en muchos países, dejando de ser una característica exclusiva de la atención sanitaria de los países de altos ingresos. De los 70.000 órganos sólidos trasplantados al año, aproximadamente 50.000 se utilizan para el reemplazo renal, y más de una tercera parte de estas operaciones se realizan en países de bajos o medianos ingresos ${ }^{1}$. 
Por otro lado, los trasplantes son coste-eficaces en muchas circunstancias, por ejemplo el trasplante renal no sólo proporciona tasas de supervivencia y una calidad de vida muy superior a la que se obtiene con otros tratamientos para la insuficiencia renal crónica terminal (hemodiálisis), sino que también es mucho menos costoso a largo plazo ${ }^{2}$.

No obstante, el trasplante de órganos sólidos plantea preocupaciones éticas. Los primeros conflictos bioéticos de fuerte impacto en la opinión pública se suscitaron a propósito de los trasplantes de corazón. Poder trasplantar órganos de unos seres humanos a otros constituyó en su momento fuente de estupefacción general y ocasión de reflexión moral y social. Cuestiones como la propia identidad, la trascendencia más allá de la muerte o el propio concepto de muerte fueron sacudidas por las nuevas técnicas y generaron un debate que, con el tiempo, se ha transformado en un estado de opinión favorable a los trasplantes. Precisamente, los trasplantes de órganos han sido también uno de los denominados problemas bioéticos que antes se han regulado legalmente. La legislación española sobre trasplantes establece una serie de principios generales que tratan de conjugar la libertad de la persona y el derecho al propio cuerpo con la solidaridad respecto a la sociedad que supone la donación de órganos. El primer requisito en el trasplante de órganos es la existencia de la finalidad terapéutica, el segundo de los requisitos se refiere a la confidencialidad y el tercero es el de gratuidad. Por otra parte, la promoción de la donación de órganos debe hacerse siempre de forma general y con carácter altruista y voluntario. Asimismo, en la normativa se resalta la obligación de los profesionales sanitarios de recibir formación continuada ${ }^{3,4}$.

Así pues, el trasplante de órganos sólidos es un tratamiento médico complejo, pero habitual en nuestro país. Este tratamiento permite que órganos, fundamentalmente provenientes de personas fallecidas, puedan reemplazar órganos enfermos de una persona en peligro de muerte, devolviéndole la salud y por tanto salvando o mejorando sustancialmente su calidad de vida.

En los últimos años se han producido avances significativos que han permitido mejorar los resultados de los trasplantes: en el mantenimiento del donante, en las técnicas quirúrgicas de extracción, en los líquidos de preservación de los órganos y en el tratamiento farmacológico (antibióticos de amplio espectro y nuevos inmunosupresores).

Estos resultados se deben evaluar en términos de supervivencia y calidad de vida.

\section{TRASPLANTE RENAL}

El trasplante renal se considera hoy en día el tratamiento de elección para aquellos enfermos que desarrollan una insuficiencia renal crónica terminal debida principalmente a la diabetes, la glomerulonefritis o la enfermedad quística. La supervivencia del injerto al año del trasplante de donante cadáver es del $90 \%$, estando la vida media del injerto en el trasplante renal de cadáver en unos 10 años.
El éxito alcanzado en el trasplante renal es el motivo de que, en la actualidad, se trasplanten más de 100.000 riñones anualmente en todo el mundo. Este mismo éxito es el que provoca que exista una lista de espera que se sitúa alrededor de las 4.000 personas en España ${ }^{5}$. Para ellas, y mientras no llega el órgano adecuado, existe la alternativa de someterse a diálisis.

En los últimos años ha cambiado el perfil del donante-receptor, con donantes y receptores de mayor edad y con enfermedades asociadas como la diabetes o la patología cardiovascular. El perfil del donante renal actual sería un hombre ( $60 \%$ donantes), con una edad media de 48,2 años, en muerte encefálica principalmente producida por un accidente cerebrovascular agudo (ACVA) $(59,4 \%)$ o un traumatismo craneoencefálico (TCE) $(29 \%)^{5}$.

Los trasplantes de órganos procedentes de donantes vivos tienen, en general, mejor supervivencia que los de cadáver, y esto es así por factores inmunológicos, por las condiciones biológicas del órgano trasplantado y por los mínimos intervalos de isquemia fría ocasionados ${ }^{6}$.

Las indicaciones de tratamiento con trasplante renal están limitadas por dos factores principales: patología extrarrenal y recidiva de la enfermedad renal, aunque esta última con matices.

En las situaciones con infección activa tenemos que descartar el trasplante renal por el riesgo que supone la inmunosupresión. Igualmente la patología asociada frecuentemente al proceso renal que acompaña o se desarrolla en un buen número de pacientes en hemodiálisis, tales como la hepatopatía crónica, afectación cardíaca, enfermedades pulmonares crónicas o arteriosclerosis generalizada hacen inviable la opción del trasplante renal. Por otro lado, la adicción a drogas impide la inclusión de pacientes en lista de espera para trasplante.

La nefropatía crónica del trasplante (NCT) constituye la causa más frecuente de pérdida tardía del injerto $^{7}$. Desde el punto de vista clínico la NCT se define como un deterioro lento y progresivo de la función renal a partir del sexto mes del trasplante. Desde el punto de vista histológico se define como la presencia de fibrosis intersticial y atrofia tubular en presencia o no de vasculopatía del trasplante. El sistema internacional de clasificación de las lesiones del aloinjerto, conocido como el de Banff, clasifica la gravedad de la NCT en tres grados de acuerdo con la extensión de la fibrosis intersticial y la atrofia tubular'.

La patogénesis de la NCT sigue siendo mal conocida. Hoy se acepta que el desarrollo y progresión de la NCT depende tanto de la respuesta aloinmune como de otros factores no inmunes. Dentro de los factores de riesgo para el desarrollo de una NCT se enumeran los siguientes: histocompatibilidad, hipersensibilización, rechazo agudo, tiempo de isquemia fría, necrosis tubular postrasplante, edad, sexo y raza del donante, tamaño del receptor, hiperlipemia, hipertensión e infección por citomegalovirus (CMV).

Hoy en día no existe ningún medicamento cuya eficacia clínica haya sido demostrada de una forma convincente, ni en la prevención ni en el tratamiento 
de la NCT. Constituyen medidas eficaces en la prevención de la NCT la existencia de un alto grado de histocompatibilidad entre el donante y el receptor, la disminución del rechazo agudo y la prevención de la nefrotoxicidad con ciclosporina o tacrolimus.

\section{Calidad de vida del paciente con trasplante renal}

El trasplante renal no supone únicamente la liberación de la diálisis para el enfermo, sino también una completa recuperación de la función renal. Así, el enfermo renal recupera su estado de salud perdido con el desarrollo de la insuficiencia renal crónica terminal y no recuperado con la diálisis. Por este motivo, todos los estudios de calidad de vida del trasplante renal ponen de manifiesto dos aspectos distintos que deben ser considerados: la mejoría en la percepción de salud del enfermo y la liberación de la diálisis y de sus restricciones dietéticas asociadas.

Probablemente por estos dos aspectos los enfermos renales prefieren el trasplante renal a la diálisis, pero además el trasplante consigue recuperar la salud, no conseguida con la diálisis.

Todos los estudios de calidad de vida del trasplante renal publicados coinciden en la mejoría significativa en la calidad del trasplante renal respecto a la diálisis, ya que éste mejora sustancialmente la calidad de vida. Los aspectos relacionados con la actividad física mejoran en un $80 \%$ de los estudios publicados; los relacionados con la actividad mental-psíquica en un $80 \%$, los sociales en un $60 \%$ y de forma global el $100 \%$ de los estudios coinciden en una mejoría sustancial $^{9,10}$.

Hay 4 aspectos relacionados con la mejoría de la calidad de vida en el paciente trasplantado que merecen ser resaltados: a) los hombres experimentan una mejoría más significativa que las mujeres en la calidad de vida después del trasplante; b) la comorbilidad asociada al paciente supone un factor determinante en la calidad de vida del trasplantado renal; c) en los pacientes de edad avanzada el trasplante renal también supone una clara mejoría en la calidad de vida respecto de la diálisis, y d) existen algunos factores directamente relacionados con el trasplante que limitan la mejoría en la calidad de vida del trasplantado renal.

El trasplante renal mejora notablemente la calidad de vida del enfermo renal respecto de la diálisis, tanto en el aspecto de actividad física como psíquica y social, o en su valoración global de calidad de vida. En general, los hombres mejoran más que las mujeres y los factores de comorbilidad asociados a la insuficiencia renal limitan la mejoría en la calidad de vida. Aspectos directamente relacionados con el trasplante renal, el tratamiento inmunosupresor y la función renal resultarán decisivos en la calidad de vida lograda después del trasplante renal $^{11}$.

\section{TRASPLANTE HEPÁTICO}

El trasplante hepático ortotópico consiste en la extirpación de un hígado enfermo y su sustitución, en la misma localización anatómica, por un hígado sano procedente de un donante cadáver o por parte del hígado procedente de un donante vivo. El trasplante hepático es hoy día el tratamiento de elección en pacientes con hepatopatías graves que no responden a otro tipo de tratamiento.

Esta terapéutica se inició en España en 1984 (Hospital de Bellvitge de Barcelona), y se han realizado cerca de 5.000 trasplantes en los 23 centros que hoy día lo practican en nuestro país. Las indicaciones más frecuentes del trasplante hepático son las cirrosis, fundamentalmente alcohólicas y víricas, seguidas de enfermedad tumoral, y cada vez es más frecuente el retrasplante por fallo crónico del injerto ${ }^{12}$.

Hasta el día de hoy se han realizado más de 50.000 trasplantes hepáticos en el mundo, con una supervivencia del paciente situada entre el 70 y el $85 \%$ al año del trasplante y entre el 60 y el $65 \%$ a los 5 años ${ }^{1}$.

Según datos de la Organización Nacional de Trasplantes (ONT) en España el 64\% de los pacientes fueron trasplantados antes de los tres meses de su inclusión en lista de espera, con una mortalidad en dicha lista del 7,5\%. La supervivencia de los pacientes y de los injertos al año del trasplante, según el registro español, es algo superior al 80 y $70 \%$, siendo a los 5 años del 70 y $60 \%$ respectivamente 5 .

Las tasas de supervivencia se relacionan con el estado general del paciente, así como con el motivo del trasplante, siendo peores los resultados en caso de trasplante hepático por insuficiencia hepática aguda grave $^{13,14}$.

Aunque esta supervivencia puede incluso incrementarse, uno de los objetivos fundamentales de los equipos de trasplante hepático es mejorar la calidad de vida de estos pacientes. Sería pues fundamental valorar, prevenir o tratar aquellas complicaciones postrasplante que provocan el daño crónico del injerto.

El daño crónico del injerto serían aquellas complicaciones que se producen después de los tres meses del implante, puesto que durante este tiempo las complicaciones se asumen como algo inherente al periodo postoperatorio. El rechazo crónico presenta una incidencia actual inferior al 5\%. El cuadro clínico corresponde a una colestasis, y la biopsia suele mostrar ductopenia importante ${ }^{15}$. En los últimos años las nuevas pautas de inmunosupresión aplicadas en ensayos clínicos de gran amplitud han logrado reducir esta complicación hasta cifras que no superan el 2-3\%. A pesar de esta tendencia favorable el rechazo ductopénico del injerto hepático es una complicación muy grave que justifica mantener un considerable grado de alerta. Debe mejorar el conocimiento de los mecanismos inmunológicos implicados y el de los factores predisponentes o favorecedores, cuyo peso específico no está aún bien determinado. Su diagnóstico debe ser temprano, sospechado en las alteraciones analíticas, pero precisa de confirmación anatomopatológica.

El tratamiento del rechazo ductopénico ha progresado en los últimos años gracias a la incorporación de nuevos inmunosupresores (ciclosporina, tacrolimus y mofetil micofenolato), que han aumentado la propor- 
ción de casos que pueden ser rescatados con tratamiento médico. A pesar de ello el rechazo crónico es una causa frecuente de disfunción grave del injerto que sólo puede ser tratado, si no hay contraindicaciones, mediante el retrasplante. En los registros nacional y europeo de trasplante el rechazo crónico justifica el $20-25 \%$ de los retrasplantes ${ }^{14,15}$.

\section{Calidad de vida del paciente con trasplante hepático}

La cuestión fundamental a la que debe responderse el médico respecto del efecto de un tratamiento sobre la calidad de vida es: ¿conseguirá este tratamiento que mi paciente se encuentre mejor? ${ }^{16}$. En el caso del trasplante hepático es evidente que mejora la supervivencia, de forma que la probabilidad de supervivencia para un paciente con cirrosis descompensada o con hepatocarcinoma se incrementa de forma notable y significativa tras el trasplante. Por tanto, los efectos beneficiosos del trasplante hepático son incuestionables. Cuando nos ceñimos a aspectos más estrictamente relacionados con la calidad de vida, la mayoría de los estudios en trasplante hepático demuestran que más de un $80 \%$ de los pacientes son capaces de desarrollar una actividad normal tras el mismo. Los niños pueden volver a la escuela y su desarrollo somático y psicológico es normal. Los pacientes adultos pueden retomar sus responsabilidades familiares, mantener una actividad social normal y algunos de ellos vuelven al trabajo ${ }^{17,18}$. Por último, a pesar de tener que recibir tratamiento inmunosupresor de por vida, los hombres pueden ser padres y las mujeres pueden quedarse embarazadas.

\section{TRASPLANTE CARDÍACO}

El trasplante cardíaco es un tratamiento complejo que en la actualidad se puede considerar una terapia consolidada y de uso rutinario en muchos de nuestros hospitales.

La insuficiencia cardíaca constituye una enfermedad de muy elevada prevalencia (1-2\% de la población general), que llega a unas cifras alarmantes en mayores de 75 años (10\%). Su frecuencia está aumentando por el incremento de la esperanza de vida en el mundo occidental y los avances en el tratamiento de la cardiopatía isquémica. La mortalidad de la insuficiencia cardíaca crónica terminal en grado funcional IV es del $50 \%$ en un año. La mortalidad a los 5 años en los hombres es del $75 \%$ y en las mujeres del $62 \%{ }^{19}$.

La supervivencia tras el trasplante cardíaco ha mejorado de manera notable, pero especialmente al reducirse la mortalidad en los primeros tres meses ${ }^{20,21}$. Este hecho refleja no solamente un mejor conocimiento de la técnica quirúrgica, de la protección miocárdica o del manejo postoperatorio, sino también de la selección de los receptores y la elección del momento idóneo para el trasplante. La selección apropiada de los receptores constituye el factor más importante para mejorar la supervivencia a corto y largo plazo $^{22}$.

En la selección del receptor se debe tener en cuenta: indicaciones, contraindicaciones y momento óptimo del trasplante.

En principio se acepta en el ámbito internacional que el paciente candidato para trasplante debe sufrir de insuficiencia cardíaca terminal con pronóstico claramente peor al del trasplante, que se encuentre muy incapacitado, sin posibilidades de tratamiento médico o quirúrgico convencional, sin fallo de otros órganos a consecuencia de la insuficiencia cardíaca ni otras enfermedades sistémicas que puedan tener un impacto negativo en la supervivencia o en la recuperación.

En la actualidad se aceptan las siguientes indicaciones para el trasplante de corazón: a) cardiopatía en fase terminal, con expectativas de vida inferior a un año; b) grado funcional III-IV (New York Heart Association [NYHA]); c) edad menor de 65 años, y d) cardiopatía no susceptible de mejoría o curación con el tratamiento médico o quirúrgico convencional.

Entre las contraindicaciones para el trasplante cardíaco se encuentran: a) edad avanzada (> 65-70 años); b) infección activa; c) enfermedad ulcerosa en fase activa; d) diabetes mellitus severa con enfermedad microvascular o de difícil control con insulina; e) obesidad mórbida o caquexia, enfermedad cerebrovascular o vascular periférica severa; f) insuficiencia pulmonar severa o bronquitis crónica severa; g) fallo renal severo (aclaramiento de creatinina $<40-50$ $\mathrm{ml} / \mathrm{min})$; h) cáncer (<3-5 años de la cirugía), e i) enfermedad mental severa.

Según el último Registro Nacional ${ }^{23}$ la mortalidad precoz (primeros 30 días tras el trasplante) ha sido del $15 \%$, con una supervivencia real al año, a los 5 y 10 años del 74, 62 y 47\% respectivamente. Las causas de mortalidad precoz han sido las siguientes: fallo agudo del injerto (35\%), fallo multiorgánico (15\%), infección $(10 \%)$ y rechazo $(6 \%)$, siendo el resto causas diversas (ACVA, hemorragia, problemas del injerto, causas pulmonares o cardiovasculares).

La amplia experiencia clínica adquirida por los grupos de trasplante cardíaco ha sido el factor determinante en la reducción de las principales complicaciones postoperatorias, la infección y el rechazo.

El rechazo en el trasplante cardíaco es frecuente, ya que la mayoría de los pacientes lo padecen en algún momento de su evolución clínica ${ }^{24}$.

La vasculopatía del injerto o cardiopatía isquémica acelerada, antes denominada rechazo crónico, constituye la tercera causa de muerte tras el trasplante cardíaco. La denervación permanente del corazón trasplantado impide la aparición de angina, por lo que esta grave complicación puede manifestarse con muerte súbita. En otras ocasiones se puede diagnosticar por la aparición de arritmias ventriculares graves, insuficiencia cardíaca congestiva o infarto silente. El $60 \%$ de los retrasplantes se indican por esta complicación. Las lesiones anatomopatológicas encontradas en este rechazo son el engrosamiento concéntrico de la íntima, la proliferación de fibroblastos y fibrosis, 
los agregados celulares rellenos de lípidos en la íntima, los depósitos de colesterol y la presencia de placas de ateroma.

La vasculopatía del injerto aparece en el $8 \%$ al año y en el 21,7\% de los casos a los 5 años del trasplante cardíaco. Se considera que la vasculopatía del injerto es un proceso inmunomediado, aunque otros factores contribuirían a modular su expresión ${ }^{25}$. Entre estos se incluyen: el sexo masculino, la edad y la hipertensión arterial en el donante y los episodios de rechazo agudo precoces, la infección por CMV, los fármacos inmunosupresores, la resistencia a la insulina, la hiperlipemia e hipertensión en el receptor.

La detección de la enfermedad vascular del injerto (EVI) se hace a través de la práctica de coronariografías rutinarias programadas de control. La frecuencia para la práctica de estos procedimientos varía de unos centros de trasplante a otros. La prevención de la EVI es uno de los principales retos en el campo del trasplante cardíaco. Al tratarse de una enfermedad inmunológica se supone que un mejor refinamiento en la inmunosupresión debería seguirse de una reducción en su incidencia ${ }^{26}$. De momento esto no ha sido demostrado. En cuanto al tratamiento, existen prácticas paliativas de revascularización que pueden aplicarse en casos seleccionados de afectación focal. Sin embargo, y dada la naturaleza difusa del proceso, el retrasplante es la única alternativa realmente definitiva en los casos evolucionados.

Aunque se sabe que la sensibilidad de la arteriografía coronaria convencional es baja y puede infraestimar la incidencia de este proceso, se sigue considerando el patrón oro para el diagnóstico del mismo. Sistemas de detección más sensibles, como la ecografía intravascular (IVUS), dan más información cualitativa y cuantitativa que la coronariografía convencional, aunque la validación de los hallazgos está todavía pendiente de establecer ${ }^{27}$.

\section{Calidad de vida del paciente con trasplante cardíaco}

La calidad de vida de estos pacientes depende fundamentalmente de la función del injerto, del tratamiento inmunosupresor y de determinados factores personales. En general, la calidad de vida tras el trasplante de corazón es buena en la mayoría de los pacientes que sobreviven al mismo. La recuperación completa lleva al menos 3-9 meses. El estado clínico preoperatorio es determinante en esta recuperación, ya que aquellos enfermos en clase funcional IV, con insuficiencia renal y/o respiratoria que requieren tratamiento farmacológico intenso u otras medidas más agresivas (contrapulsación, asistencia ventricular, etc.) precisan de un periodo de tiempo más prolongado para lograr una recuperación clínica y funcional.

La calidad de vida puede mejorarse progresivamente con una adecuada y precoz rehabilitación física, familiar, social y laboral. El trasplante de corazón ofrece una buena calidad de vida en el $75 \%$ de los supervivientes, pudiendo disfrutar completamente de su vida familiar, reincorporándose a la actividad laboral y deportiva, así como viajar sin grandes limitaciones $^{20,21}$.

\section{TRASPLANTE PULMONAR}

El trasplante pulmonar ha evolucionado considerablemente en los últimos 20 años y actualmente constituye una opción terapéutica que se debe considerar en pacientes con enfermedad pulmonar avanzada.

En 1983 el grupo de Toronto realizó el primer trasplante unilateral con éxito a un paciente de 58 años afecto de fibrosis pulmonar, quien sobrevivió a la intervención con buena calidad de vida durante más de 5 años. En los años noventa se consolida el trasplante pulmonar como opción terapéutica y, según los datos del Registro Internacional ${ }^{21}$, el número de trasplantes fue incrementándose progresivamente, hasta estabilizarse en los últimos años en relación con la consolidación del número de donantes.

Desde su aplicación inicial en un paciente con fibrosis pulmonar las indicaciones de trasplante se han ido ampliando progresivamente, y actualmente se considera que cualquier enfermedad pulmonar en fase final es tributaria de trasplante pulmonar, siempre que no exista una contraindicación para el mismo $^{28}$.

Las indicaciones generales de trasplante pulmonar son las siguientes: edad < 65 años, enfermedad pulmonar avanzada muy sintomática (clase funcional III/IV), esperanza de vida inferior a dos años y ausencia de contraindicaciones.

Las contraindicaciones generales de trasplante pulmonar son: a) disfunción de órganos vitales como riñón, corazón, hígado y sistema nervioso central; b) infección por el virus de la inmunodeficiencia humana (VIH) (contraindicación relativa); c) enfermedad maligna activa en los últimos 2-5 años; d) serología HbsAg positiva; e) serología virus $\mathrm{C}$ positiva con evidencia de enfermedad hepática.

En cuanto a las indicaciones específicas de trasplante, se diferencian 4 grupos de enfermedades:

1. Las enfermedades supurativas, como la fibrosis quística y las bronquiectasias de otra etiología, se caracterizan por una infección bronquial crónica que destruye y dilata progresivamente la luz bronquial.

2. En las enfermedades intersticiales, como la fibrosis idiopática, se produce una sustitución difusa del parénquima pulmonar por fibras de colágeno, que destruyen progresivamente los espacios alveolares.

3. En las enfermedades obstructivas se incluyen, fundamentalmente, el enfisema y la bronquitis crónica.

4. En las enfermedades vasculares se produce un incremento de la resistencia a la circulación pulmonar por una reducción del lecho vascular pulmonar de carácter idiopático en la hipertensión pulmonar primaria, por obstrucción crónica secundaria a tromboembolismos pulmonares de repetición, o a un aumento del flujo sanguíneo pulmonar en las malformaciones congénitas.

Según el Registro Internacional ${ }^{21}$, la causa más frecuente de trasplante unilateral es el enfisema, con más de la mitad de los casos, considerando conjunta- 
mente el enfisema del fumador con el asociado a un déficit congénito de alfa-1-antitripsina. Tras el enfisema, la fibrosis pulmonar, con mucha menor frecuencia, es la segunda indicación de trasplante unilateral. La causa más frecuente de trasplante bilateral es la fibrosis quística, seguida del enfisema.

La realización de un trasplante pulmonar unilateral o bilateral viene condicionada fundamentalmente por la enfermedad de base del paciente y la disponibilidad de órganos. Las posibilidades que se pueden considerar son el trasplante unilateral, el trasplante bilateral y el trasplante conjunto de ambos pulmones con el corazón, en lo que se denomina el trasplante cardíaco y pulmonar en bloque. El trasplante unilateral es el procedimiento adecuado en las enfermedades intersticiales como la fibrosis pulmonar, y en los pacientes con enfermedad obstructiva, en los que también puede realizarse un trasplante bilateral. El trasplante bilateral es el procedimiento indicado en pacientes con enfermedad supurativa para evitar que el injerto se contamine con las secreciones purulentas procedentes del pulmón nativo. En los pacientes que presentan de forma conjunta una enfermedad pulmonar y cardíaca irreversible, y en la hipertensión pulmonar, el procedimiento indicado es el trasplante cardiopulmonar en bloque.

En cuanto a la supervivencia, los datos del Registro Internacional $^{21}$ y los de nuestro país son superponibles: $70 \%$ al primer año, $58 \%$ al tercer año y cercana al $50 \%$ a los 5 años. La causa principal de mortalidad precoz (0-30 días) es el fallo primario del injerto producido como consecuencia del daño que sufre la membrana alveolocapilar tras la isquemia y la posterior reperfusión pulmonar. Las infecciones son siempre una causa importante de mortalidad, especialmente en el primer mes y durante el primer año, cuando la inmunosupresión es mayor. A partir del primer año la bronquiolitis obliterante, caracterizada por obstrucción y destrucción progresiva de la vía aérea, como forma clínico-patológica de rechazo crónico, es la principal causa de mortalidad.

En los pacientes que sobreviven los resultados funcionales son muy buenos y se alcanzan cifras de capacidad vital y de volumen espiratorio forzado en el primer segundo (FEV1) superiores al 50\% en el trasplante unilateral, y cercanas o superiores al $100 \%$ en el trasplante bilateral. La función pulmonar se mantiene estable a lo largo del tiempo hasta el desarrollo del rechazo crónico. Distintos estudios sobre la calidad de vida han demostrado que los receptores que sobreviven tienen mejor calidad de vida que los candidatos, y que las mejoras se mantienen en el tiempo hasta el desarrollo del rechazo crónico ${ }^{29-31}$.

\section{TRASPLANTE DE PÁNCREAS}

La diabetes insulinodependiente constituye uno de los mayores problemas sanitarios del mundo occidental. La diabetes tipo 1 tiene, en gran parte de los pacientes, un carácter devastador como consecuencia, fundamentalmente, de las complicaciones vasculares macro y microangiopáticas (insuficiencia renal, cegue- ra, infarto de miocardio, amputaciones, etc.). Debido a ello estos pacientes presentan un grave deterioro tanto de la calidad como de la expectativa de vida.

De las distintas opciones terapéuticas el trasplante de páncreas es la única alternativa que normaliza de forma sostenida la homeostasis de la glucosa, liberando a los diabéticos de la dependencia insulínica y de las restricciones físicas y dietéticas; más controvertido es su efecto sobre la evolución de las complicaciones secundarias ${ }^{32}$.

La necesidad de inmunosupresión, para la prevención del rechazo, hace que el trasplante de páncreas deba restringirse a los pacientes en los que las complicaciones de la diabetes son, o pueden llegar a ser, más severas que los potenciales efectos indeseables de la medicación antirrechazo. Idealmente, el trasplante de páncreas debería realizarse antes de la aparición de las complicaciones secundarias de la diabetes. Sin embargo, la dificultad para predecir qué diabéticos las van a desarrollar hace que prácticamente todos sean trasplantados ya en fase de complicaciones y la mayoría en fase de insuficiencia renal terminal, en los que al ser necesario el trasplante de riñón la inmunosupresión es inevitable.

\section{Trasplante de páncreas asociado al trasplante de riñón}

En los pacientes diabéticos con insuficiencia renal terminal el trasplante de páncreas puede hacerse de forma simultánea al trasplante renal, o después, una vez estabilizada la función del injerto renal. El trasplante simultáneo tiene la ventaja de que los dos órganos proceden del mismo donante y sólo necesita un acto quirúrgico y una pauta de inmunosupresión.

\section{Trasplante de páncreas aislado}

En pacientes no urémicos se consideran candidatos al trasplante de páncreas los afectos de retinopatía proproliferativa, nefropatía diabética, diabetes hiperlábil o dolor intratable debido a neuropatía diabética.

A pesar de los innegables avances en los resultados del trasplante de páncreas, sigue viva la controversia sobre su capacidad para modificar favorablemente el curso de las complicaciones secundarias de la diabetes.

La experiencia clínica evidencia que el trasplante de páncreas puede frenar el desarrollo de la nefropatía diabética y, en el caso del trasplante simultáneo con riñón, evitar que aparezca en el paciente trasplantado la neuropatía diabética, siendo más controvertido el efecto de éste sobre la retinopatía.

\section{Calidad de vida del paciente con trasplante de páncreas}

La calidad de vida del paciente diabético mejora notablemente tras el trasplante de páncreas ${ }^{33-35}$. Los múltiples estudios existentes emplean diferentes herramientas para evaluar la calidad de vida, como SF-36, 
Karnofsky Index, Nottingham Health Profile, etc. Habitualmente la información se recoge antes del trasplante, 6 y 12 meses después de haberse efectuado y una vez por año. Es un hecho evidente que en todos los estudios se objetiva una marcada mejoría tras el trasplante.

En estudios realizados con el Registro Internacional $^{36,37}$, con un gran número de pacientes (más de 23.000 trasplantes de páncreas) correspondientes a diversas instituciones durante un periodo de 15 años, se objetivó una significativa mejoría en los tres grupos de sujetos analizados (trasplante doble simultáneo, trasplante de páncreas después del renal funcionante y trasplante de riñón aislado). La conclusión de dichos estudios es que, tras el trasplante de páncreas, se obtiene:

1. Rehabilitación de una vida normal, con bienestar físico, psíquico y social, con actividades diarias normales y autopercepción de normalidad.

2. Mejoría global de la calidad de vida con percepción de buena salud y control sobre su destino.

3. Muy pocas restricciones y, por tanto, posibilidad de aumentar la sensación de bienestar e independencia.

4. La mayoría encuentra más fácil el control de la inmunosupresión que el control de la terapia con insulina.

5. El número de ingresos hospitalarios desciende de 2,4 por año en el primer año postrasplante a 0,6 en el cuarto año.

6. Tras el trasplante el $80 \%$ de los pacientes no tiene limitación de la actividad, comparado con la situación previa en la que solamente el $30 \%$ no tenía esta limitación.

\section{Declaración de conflicto de intereses}

Los autores han declarado no tener ningún conflicto de intereses.

\section{BIBLIOGRAFÍA}

1. UNOS (United Network for Organ Sharfing). Disponible en: http://www.unos.org

2. Consejería de Salud de la Junta de Andalucía. Proceso asistencial integrado. Tratamiento sustitutivo de la insuficiencia renal crónica: diálisis y trasplante renal. Junta de Andalucía; 2005.

3. Ley de 27 Octubre de 1979, núm. 30/79. BOE de 6 noviembre de 1979 (núm. 266). p. 2655.

4. RD 2070/1999. BOE de 4 enero 2000 (núm. 3). p. 179-90.

5. ONT (Organización Nacional de Trasplantes). Disponible en: http://www.ont.es

6. Ortuño J. Ventajas e inconvenientes del trasplante renal de vivo. Nefrología. 1994;4:272-9.

7. Carpenter CB. Long-term failure of renal transplants: adding insult of injury. Kidney Int. 1995;48 Suppl 50:40-4.

8. Seleck Azelsevira B, Anner B, Benediktssonlt, Colvin RB. International standarization of nomenclature and criteria for the histological diagnosis of renal allograft rejection: the banff classification of kidney transplant pathology. Kidney Int. 1993;44:411-2.

9. Manu MA, Radulescu S, Harza M, Manu R, Capsa D, Sinescu I. Quality of life assessed by SF-36 health survey in renal transplant patients. Tansplant Proc. 2001;33:1927-8.

10. Nielens H, Lejeune TM, Lalaoui A, Squifflet JP, Pirson Y, Goffin E. Increase of physical activity level after successful renal transplantation: a 5 year follow-up study. Nephrol Dial Transplant. 2001;16:134-40.
11. Ortega F, Rebollo P, coordinadores. Calidad de vida relacionada con la salud de pacientes receptores de un trasplante de órgano sólido. Barcelona: Ediciones Mayo; 2006.

12. Matesanz R. Presente y futuro de los trasplantes en España. Rev Clin Esp. 1995;195:203-5.

13. Lake JR. Changing indications for liver transplantation. Gastroenterol Clin North Am. 1993;22:213-29.

14. Registro español de trasplante hepático. Novena memoria de resultados. 1984-2006. Disponible en: http://www.ont.es

15. Backman L, Gibbs J, Levy M, McMillan R, Holman M, Husberg B, et al. Causes of late graft loss after liver transplantation. Transplantation. 1993;55:1078-82.

16. Guyatt GH, Naylor CD, Juniper E, Heyland DK, Jaeschke R, Cook DJ. Users' guides to the medical literature. XII. How to use articles about health-related quality of life. Evidence-Based Medicine Working Group. JAMA. 1997;277:1232-7.

17. Lundgren M, Kristiansson M, Ericzon BG, Eleborg L. Improved quality of life after liver transplantation. Transplant Proc. 1994;26:1779.

18. Tarter RE, Switala J, Arria A, Plail J, Van Thiel D. Quality of life before and after orthotopic hepatic transplantation. Arch Intern Med. 1991;151:1521-6.

19. Concha M, Arizon JM, Crespo MG, Lloréis R. Trasplante cardíaco. Conferencia de Consenso Nacional. En: Informes y Documentos de Consenso promovidos por la Organización Nacional de Trasplantes y la Comisión de Trasplantes del Consejo Interterritorial del Sistema Nacional de Salud. 1st ed. Madrid: Editorial Complutense; 2000. p. 187-272.

20. Hosenpund JD, Bennett LE, Keck BM, Boucek MM, Novick RJ. The Registry of the International Society for Heart and Lung Transplantation: Seventeenth Official Report 2000. J Heart Lung Transplant. 2000;19:909-31.

21. Taylor DO, Edwards LB, Boucek MM, Trulock EP, Aurora P, Christie J, et al. Registry of the International Society for Heart and Lung Transplantation: twenty-fourth official adult heart transplant report 2007. J Heart Lung Transplant. 2007;26:769-81.

22. Mehra MR, Kobashigawa J, Starling R, Russell S, Uber PA, Parameshwar J, et al. Listing criteria for heart transplantation: Society for Heart and Lung transplantation guidelines for the care of cardiac transplant candidates 2006. J Heart Lung Transplant. 2006;25:1024-42.

23. Almenar Bonet L. Registro Español de Trasplante Cardiaco. XVII Informe Oficial (1984-2005). Rev Esp Cardiol. 2006;59:1283-91.

24. Billingham ME, Cary NRB, Hammond ME, Kemnitz J, Marboe C, McCallister HA, et al. A working formulation for the standarization of nomenclature in the diagnosis of heart and lung rejection: heart rejection study group. J Heart Transplant. 1990;9: 587-93.

25. Yeung AC, Anderson T, Meredith I, Uehata A, Ryan TJ, Selwyn AP, et al. Endothelial dysfunction in the development and detection of transplant coronary artery disease. J Heart Lung Transplant. 1992;11(3 Pt 2):S69-73.

26. Renlund DG, Gopinathan SK, Kfoury AG, Taylor DO. Mycophenolate mofetil (MMF) in heart transplantation: rejection prevention and treatment. Clin Transplant. 1996;10 1 Pt 2:136-9.

27. St Goar FG, Pinto FJ, Alderman EL, Valantine HA, Schroeder JS, Gao SZ, et al. Intracoronary ultrasound in cardiac transplant recipients. In vivo evidence of "angiographically silent" intimal thickening. Circulation. 1992;85:979-87.

28. American Thoracic Society. International guidelines for the selection of lung transplant candidates. Am J Respir Crit Care Med. 1998;158:335-9.

29. Lanuza DM, Lefaiver $\mathrm{CH}, \mathrm{Mc}$ Cabe M, Farcas GA, Garrity E Jr. Prospective study of functional status and quality of life before and after lung transplantation. Chest. 2000;118:115-22.

30. Smeritschnig B, Jaksch P, Kocher A, Seebacher G, Aigner C, Mazhar S, et al. Quality of life after lung transplantation: a cross-sectional study. J Heart Lung Transplant. 2005;24:474-80.

31. Limbos MM, Joyce DP, Chan CK, Kesten S. Psychological functioning and quality of life in lung transplant candidates and recipients. Chest. 2000;118:408-16.

32. Organización Nacional de Trasplantes. Documento de Consenso sobre Trasplante de Páncreas e Islotes. Madrid: Aula Médica Ediciones; 2005. 
33. Gross CR, Limwattananon C, Matthees BJ. Quality of life after pancreas transplantation: a review. Clin Transplant. 1998;12: $351-61$.

34. Zehrer CL, Gross CR. Quality of life of pancreas transplant recipients. Diabetología. 1991;34 Suppl 1:145-9.

35. Fioretto P, Steffes MW, Sutherland DER, Goetz FC, Mauer M. Reversal of lesions of diabetic nephropathy after pancreas transplantation. N Engl J Med. 1998;339:69-75.
36. Gruessner AC, Sutherland DE. Report for the international pancreas transplant registry-2000. Transplant Proc. 2001;33: $1643-6$.

37. Gruessner AC, Sutherland DE. Pancreas transplant outcomes for United States (US) and non-US cases as reported to the United Network for Organ Sharing (UNOS) and the International Pancreas Transplant Registry (IPTR) as of June 2004. Clin Transplant. 2005; 19:433-55. 